\title{
High-accuracy wave field reconstruction: decoupled \\ inverse imaging with sparse modeling of phase and

\author{
amplitude
}

\author{
Vladimir Katkovnik and Jaakko Astola
}

We apply a nonlocal adaptive spectral transform for sparse modeling of phase and amplitude of a coherent wave field. The reconstruction of this wave field from complex-valued Gaussian noisy observations is considered. The problem is formulated as a multi-objective constrained optimization. The developed iterative algorithm decouples the inversion of the forward propagation operator and the filtering of phase and amplitude of the wave field. It is demonstrated by simulations that the performance of the algorithm, both visually and numerically, is the current state-of-the-art. (C) 2011 Optical Society of America

OCIS codes: 090.1995, 100.1830 100.3010, 100.3190

Department of Signal Processing, Tampere University of Technology, P.O. Box 527, FI-33101 Tampere, Finland 


\section{${ }^{*}$ Corresponding author: vladmir.katkovnik@tut.fi}

\section{Introduction}

Let us consider a wave field reconstruction in the following setup. A coherent complex-valued wave field propagates from an object plane to a parallel image plane. This free space forward propagation is modeled by the Rayleigh-Sommerfeld diffraction integral. The problem is to reconstruct the complex-valued wave field at the object plane from complex-valued observations given at the image plane.

Numerical backward propagation operators derived from the Rayleigh-Sommerfeld integral are the conventional tools for the problem $[1,2]$. However, it has long been recognized that the forward propagation cannot be compensated properly by these backward operators. Typi-

cally, obtained reconstructions are rather blurred and exhibit pronounced "waves", "wiggles" and "ringing". The optical diffraction is one of the main sources of these artifacts.

Mathematically, free space forward propagation operators, mapping an object wave field to a finite-size sensor at the image plane, are ill-posed. It follows that no linear operators exist which are inverse to the forward propagation. It is a fundamental reason why it is not possible to compensate the forward propagation precisely.

In this paper, we propose a novel inverse imaging technique with the main motivation to wipe out the mentioned typical artifacts, filter noise (if observations are noisy) and to achieve crisp imaging. A special sparse modeling is developed for phase and amplitude of the wave field at the object plane in order to overcome the loss of information due to the ill-posedness of the forward propagation operators. This flexible and data adaptive modeling relies on the recent progress in sparse imaging $[3,4]$. 
Being in line with the general formalism of sparse imaging, we develop an original approach and algorithm which are different from the main stream of sparse imaging in the following aspects.

First, to deal with complex-valued wave fields comprehensively, we use separate sparse modeling for phase and amplitude.

Second, assuming that phase and amplitude can be spatially varying, continuous or discontinuous, we apply for their modeling (approximation) novel powerful dictionaries known as $B M 3 D$-frames [5-7]. These frames are derived from the analysis and synthesis procedures of Block Matching 3D (BM3D) filter, a well-established tool for imaging denoising and other imaging problems [8-10].

Third, while the conventional sparse imaging uses a single-objective optimization, contrary to it, the algorithm developed in this paper is based on a multi-objective optimization with two objective functions minimized alternatingly. The algorithm searches for fixed-point giving a balance between two quality measures defined by these objective functions. This vector optimization, originated in $[6,7]$, decouples the inversion and filtering operations.

The rest of the paper is organized as follows. In Section 2 we present a basic formalism of the used wave field modeling. The frame based spectral representations for phase and amplitude are introduced in Section 3. Constrained variational formulations for phase and amplitude reconstruction are considered in Section 4. The developed algorithm is presented in Section 5. Numerical experiments demonstrating a very good performance of the algorithm are discussed in Section 6. The mathematical derivation of the main steps of the algorithm is given in Appendix. Some preliminary results concerning the proposed approach and algorithm have been presented at [11]. 


\section{Image formation}

Let $u_{0}(x)$ and $u_{z}(x)$ denote complex-valued wave field distributions at the object and image planes, respectively, given in the lateral coordinates $x \in \mathbb{R}^{2}$. In discrete modeling the continuous argument $x$ is replaced by the digital one with a corresponding replacement of all continuous functions by their discrete counterparts: $u_{0}(x) \rightarrow u_{0}[k], u_{z}(x) \rightarrow u_{z}[k]$ with $2 D$ integer argument $k$. This discretization assumes that wave field distributions are pixelated, i.e. pixel-wise invariant.

In what follows we use a vector-matrix notation with $N_{1} \times N_{2}$ images given as vectors in $\mathbb{C}^{n}, n=N_{1} N_{2}$, where $\mathbb{C}^{n}$ stands for the space of complex-valued vectors of length $n$. These vectors are obtained from the standard matrix representation for images by concatenating the columns of these matrices. We use bold lower case characters for these vectors. Thus, $\mathbf{u}_{0}$ and $\mathbf{u}_{z}$ are vectorial representations for $u_{0}[k]$ and $u_{z}[k]$.

The forward propagation of the wave field from the object plane with a distribution $\mathbf{u}_{0}$ gives a distribution $\mathbf{u}_{z}$ at the image plane as

$$
\mathbf{u}_{z}=\mathbf{A}_{z} \mathbf{u}_{0}
$$

where $\mathbf{A}_{z}$ is a generic notation for a discrete forward propagation operator (complex-valued $n \times n$ matrix, $\left.\mathbf{A}_{z} \in \mathbb{C}^{n \times n}\right)$.

Eq. (1) corresponds to the convolution of the object distribution $\mathbf{u}_{0}$ with the diffraction kernel of the wave field propagation operator $\mathbf{A}_{z}$. Depending on the used discretization of the Rayleigh-Sommerfield integral the operator $\mathbf{A}_{z}$ in Eq. (1) can be: convolutional $[2,12]$; angular spectrum decomposition (ASD) [1,2]; discrete diffraction transform (DDT) given in the image (M-DDT) [13] or Fourier (F-DDT) [14] domains. The latter DDT models, derived 
for the Fresnel approximation of the Rayleigh-Sommerfield integral, enable the precise pixelto-pixel mapping of any pixelated $\mathbf{u}_{0}$ to the corresponding $\mathbf{u}_{z}$.

According to Eq. (1) the observation model with an additive Gaussian noise in the image (sensor) plane takes the form

$$
\mathbf{y}=\mathbf{A}_{z} \mathbf{u}_{0}+\sigma \varepsilon
$$

where the noise $\varepsilon=\varepsilon_{1}+j \varepsilon_{2}, \varepsilon \in \mathbb{C}^{n}$, is complex-valued, circular, standard Gaussian, i.e. real and imaginary parts of $\varepsilon$ are i.i.d. and $\varepsilon_{1}, \varepsilon_{2} \sim \mathcal{N}\left(\mathbf{0}, \mathbf{I}_{n \times n}\right), \sigma$ is the standard deviation.

The "naive" inverse of $\mathbf{A}_{z}$ gives $\hat{\mathbf{u}}_{0}=\mathbf{A}_{z}^{-1} \mathbf{y}$ as a solution of (2). If $\mathbf{A}_{z}$ is ill-posed, this solution is highly sensitive to noise and round errors. The least squares and maximum likelihood provide valuable regularized alternatives to this solution (e.g. [15]). Sparse imaging is a special regularization technique where the regularization is introduced through a sparse image modeling.

It is assumed in sparse imaging that there exists a basis consisting of a small number of items where $\mathbf{u}_{0}$ can be represented exactly or approximately with a very good accuracy. This ideal basis is a priori unknown and selected from a given set of potential bases (dictionary or dictionaries). Sparse imaging can be viewed as a parametric approximation of signals with an adaptive basis selection, one of the classical topics in statistics. The modern popularity and success of sparse imaging are due to the novel attractive theory, the efficient algorithms and the evidence that the developed formalism fits perfectly to many important applications.

The compressive (or compressed) sensing (CS ) is one of the fields where the sparse imaging techniques are efficient. In CS the object distribution $\mathbf{u}_{0}$ is reconstructed from subsampled data. A total number of available observations can be smaller (much smaller) 
than size of $\mathbf{u}_{0}$. It is proved in CS that the perfect reconstruction of $\mathbf{u}_{0}$ from the subsampled data can be achieved for sparse object distributions [16-18].

The reconstruction of $\mathbf{u}_{0}$ from the observation $\mathbf{y}$ in (2) can be treated as a special type of the CS problems because due to the ill-posedness of $\mathbf{A}_{z}$ a number of linear independent items in the vector $\mathbf{A}_{z} \mathbf{u}_{0}$ is smaller (even much smaller) than length of this vector. Thus, an illposed blur operator results in the effects which are equivalent to subsampling of observations in CS.

Recently in optics, sparse imaging has become a subject of multiple applications in the context of CS. Complex-valued signals and operators are distinctive features of this development. Basic facts of the corresponding CS theory, algorithms, simulations as well as experimental demonstrations can be found [19], where CS is used for sub-wavelength imaging overcoming the diffraction limitations. The penalization (regularization) for variational image reconstructions in [19] are formulated using the $l_{0^{-}}$and $l_{1}$-norms for spectra of object representations.

We wish to mention also few works on CS which are relevant to our paper. A slice-by-slice reconstruction of a 3D object from the holographic data is considered in [20] and [21]. In [22] a maximum likelihood approach is developed for estimation of the object density from $2 \mathrm{D}$ scattered fully developed speckle field measurements. The total variation (TV) penalization is exploited as a regularization tool in this inverse imaging. A compressive Fresnel holography in [23] is developed using a combination of two types of the penalties $l_{1}$-norm and TV.

It is assumed in the above cited papers that the wave field is modeled as a linear combination of some basic functions. Phase and amplitude signals are mixed together in these approximations. In particular, in [19] the phase issue appears only as a sign of the real-valued 
wave field distribution. The reconstruction of a full range of phase values is not considered.

In our opinion, the only way to the high-accuracy is a nonlinear modeling of the wave field with separate approximations for phase and amplitude.

At this point we refer to the work [24], where a strong improvement in CS hyperspectral imaging is demonstrated due to the quadratic penalization for phase used jointly with the TV penalty for the complex valued wave field. This is a good example of an efficient penalization separate for phase and amplitude.

In our paper we develop a general approach to the problem using a high-order adaptive approximation for phase and amplitude.

\section{Sparse modeling for phase and amplitude}

\section{A. Frames for phase and amplitude}

Sparse image approximations can be given in two different synthesis and analysis forms, respectively, as follows

$$
\mathrm{Y}=\Psi \boldsymbol{\theta}
$$

and

$$
\theta=\Phi Y
$$

Here, $\mathbf{Y} \in \mathbb{R}^{n}, \boldsymbol{\theta} \in \mathbb{R}^{m}$, and $\boldsymbol{\Psi}$ and $\boldsymbol{\Phi}$ are transform matrices of $n \times m$ and $m \times n$, respectively. The vector $\boldsymbol{\theta}$, usually called spectrum, gives the parameters for the parametric approximation

of the image $\mathbf{Y}$ as $\mathbf{Y}=\boldsymbol{\Psi} \boldsymbol{\theta}$. Thus, $\mathbf{Y}=\sum_{j=1}^{m} \boldsymbol{\Psi}_{j} \cdot \boldsymbol{\theta}_{j}$, where $\boldsymbol{\Psi}_{j}$ are the columns of the matrix $\boldsymbol{\Psi}$, and $\boldsymbol{\theta}_{j}$ are the items of the vector $\boldsymbol{\theta}$.

If the synthesis defines the image provided that the spectrum is given, $\mathbf{Y}=\boldsymbol{\Psi} \boldsymbol{\theta}$, the 
analysis defines the spectrum corresponding to a given image as $\boldsymbol{\theta}=\boldsymbol{\Phi} \mathbf{Y}$.

It is recognized that overcomplete representations for $\mathbf{Y}$ with $m \gg n$ and linearly depen$\operatorname{dent} \boldsymbol{\Psi}_{j}$ form a much more powerful tool for advanced imaging than the classical orthonormal bases, where $m=n$. The concept of frame is a generalization of the classical bases developed for overcomplete (synthesis and analysis) representations with linearly dependent approximating functions (e.g. [25]).

There are special links between the analysis and synthesis frames. The requirement, $\boldsymbol{\Psi} \cdot \mathbf{\Phi}=\mathbf{I}_{n \times n}$, where $\mathbf{I}_{n \times n}$ is the $n \times n$ identity matrix, enables a perfect reconstruction of any $\mathbf{Y}$ from the corresponding spectrum $\boldsymbol{\theta}$, indeed $\mathbf{Y}=\boldsymbol{\Psi} \boldsymbol{\theta}=\boldsymbol{\Psi} \cdot \boldsymbol{\Phi} \mathbf{Y}=\mathbf{Y}$. For the so-called tight frames, $\boldsymbol{\Phi}^{T} \cdot \boldsymbol{\Phi}=\mathbf{I}_{n \times n}$ and $\boldsymbol{\Psi}=\boldsymbol{\Phi}^{T}[25]$.

For details and applications of overcomplete, in particular, frame based modeling for imaging we refer to the recent books [3] and [4].

Modeling of the object wave field $\mathbf{u}_{0}$ lies at the core of variational approaches to wave field imaging. A complex-valued $\mathbf{u}_{0}$ requires distinct modeling for amplitude and phase. For instance, for the phase modulation the object may have an invariant amplitude and a varying unknown phase to be reconstructed. Contrary to it the amplitude modulation assumes that a phase is invariant and amplitude variations are of interest.

In this paper we apply frames for a sparse modeling of both phase (angle) and amplitude (modulus) of $\mathbf{u}_{0}$. The following equations link amplitude and phase with the corresponding transform (spectrum) representations:

$$
\begin{aligned}
& \operatorname{abs}\left(\mathbf{u}_{0}\right)=\boldsymbol{\Psi}_{a} \boldsymbol{\theta}_{a}, \operatorname{angle}\left(\mathbf{u}_{0}\right)=\boldsymbol{\Psi}_{\varphi} \boldsymbol{\theta}_{\varphi}, \\
& \boldsymbol{\theta}_{a}=\boldsymbol{\Phi}_{a} \cdot \operatorname{abs}\left(\mathbf{u}_{0}\right), \boldsymbol{\theta}_{\varphi}=\boldsymbol{\Phi}_{\varphi} \cdot \operatorname{angle}\left(\mathbf{u}_{0}\right),
\end{aligned}
$$


where $\boldsymbol{\theta}_{a}$ and $\boldsymbol{\theta}_{\varphi}$ are vectors of the amplitude and phase spectra. The modulus and angle operations applied to vectors in (5)-(6) are elementwise. Thus abs $\left(\mathbf{u}_{0}\right)$ and angle( $\left.\mathbf{u}_{0}\right)$ are the vectors of amplitude and phase values.

The frame synthesis and analysis matrices $\boldsymbol{\Psi}_{a}, \mathbf{\Phi}_{a}, \boldsymbol{\Psi}_{\varphi}, \mathbf{\Phi}_{\varphi}$ are shown with the indices $a$ and $\varphi$ for amplitude and phase, respectively.

Eqs. (5) define the synthesis giving amplitude $\left(\operatorname{abs}\left(\mathbf{u}_{0}\right)\right)$ and phase $\left(\operatorname{angle}\left(\mathbf{u}_{0}\right)\right)$ from the amplitude and phase spectra $\boldsymbol{\theta}_{a}$ and $\boldsymbol{\theta}_{\varphi}$. On the other hand, the analysis Eqs. (6) give the spectra for amplitude and phase of $\mathbf{u}_{0}$.

The $l_{0}$-norm of the vector $\boldsymbol{\theta}$, denoted by $\|\boldsymbol{\theta}\|_{0}$, is defined as a number of nonzero elements of the vector. The $l_{1}-$ norm of $\boldsymbol{\theta}$ is defined as the sum of the absolute values of all items of the vector, $\|\boldsymbol{\theta}\|_{1}=\sum_{s}\left|\boldsymbol{\theta}_{s}\right|$. Both these norms are used in order to characterize sparsity of approximation. A smaller value of the norm means a higher sparsity of approximation.

It is known, that the variational image reconstructions using the $l_{0^{-}}$and $l_{1}$-norms as penalties, provided some assumptions, give results that are close to each other. This is an important fact, because it allows to replace the non-convex $l_{0}$-norm by the convex $l_{1}$-norm in many variational settings (e.g. $[3,4])$.

In our approach the sparsity is evaluated separately for phase and amplitude by the $l_{p^{-}}$ norms, $\left\|\boldsymbol{\theta}_{\varphi}\right\|_{p}$ and $\left\|\boldsymbol{\theta}_{a}\right\|_{p}$, where $p=0,1$ for the $l_{0}-$ and $l_{1}$-norms, respectively. Overall, the main intention is to find sparsest (shortest) models for phase and amplitude with smallest values of the $l_{p}$-norms. 


\section{B. BM3D-frames}

Recently, within the framework of nonlocal patch-wise image modeling, a family of the BM3D algorithms has been developed for imaging. BM3D is a nonlocal adaptive technique based on high-order groupwise models defined in the 3D transform domain. Below we briefly recall the basic steps of the BM3D filtering which can be split into three stages $[8,9]$.

1. Analysis. Similar image blocks (patches) are collected in groups in order to obtain highly correlated data. The blocks in each group are stacked together to form a 3-D data array, which is decorrelated using an invertible 3-D transform.

2. Processing. 3-D group spectra obtained from 3-D data arrays are filtered by thresholding.

3. Synthesis. Filtered spectra are inverted providing estimates for each block in the group. These blockwise estimates are returned to their original positions in the image, and the final image estimate is aggregated by weighted averaging all of the obtained block-wise estimates.

It is shown in $[5,6]$ that the analysis and synthesis developed in the BM3D filter allow the corresponding frame analysis and synthesis interpretation. The BM3D analysis and synthesis operators can be given in the matrix form (3)-(4), linking the image $\mathbf{Y} \in \mathbb{R}^{n}$ and the groupwise 3D spectrum vector $\boldsymbol{\theta} \in \mathbb{R}^{m}$. The rows of the analysis matrix $\boldsymbol{\Phi}$ constitute a frame in $\mathbb{R}^{n}$, and the columns of the synthesis matrix $\Psi$ constitute a frame in $\mathbb{R}^{n}$ dual to $\boldsymbol{\Phi}$. In the explicit form, these BM3D-frames are presented in [7]. It is proved that the BM3Dframes are non-tight. It follows that the matrices $\boldsymbol{\Phi}$ and $\boldsymbol{\Psi}$ do not define each other, as it is 
for the tight frames, and both of them should participate in image reconstruction.

Once BM3D groups are defined, the operators $\boldsymbol{\Phi}, \boldsymbol{\Phi}^{T}, \boldsymbol{\Psi}$ and $\boldsymbol{\Psi}^{T}$ can be calculated efficiently since all of them perform groupwise separable 3-D transforms. To build the groups the block matching (grouping) procedure from [8] is used. The $B M 3 D$-frames are nonlocal and data adaptive, which make them quite different from the other popular frames used for image modeling.

It is demonstrated in [6] and [7], that the BM3D-frames give extraordinary good results for image deblurring problem. In this paper we apply these advanced BM3D-frames for modeling phase and amplitude of $\mathbf{u}_{0}$ as it is shown in Eqs. (5) and (6).

\section{Variational formulation of wave field reconstruction}

\section{A. Single-objective optimization}

One usual approach is to determine a restored image by minimizing a single objective function consisting of a fidelity (residual) and regularization terms. For the considered problem the objective function can be given in the form:

$$
\frac{1}{\mu}\left\|\mathbf{y}-\mathbf{A}_{z} \cdot \mathbf{u}_{0}\right\|_{2}^{2}+\tau_{a} \cdot\left\|\boldsymbol{\theta}_{a}\right\|_{p}+\tau_{\varphi} \cdot\left\|\boldsymbol{\theta}_{\varphi}\right\|_{p}
$$

where $\|\cdot\|_{2}^{2}$ stands for the Euclidean norm, $\mu=2 \sigma^{2}$, and the $l_{p}$-norms define regularization terms for phase and amplitude taken with parameters $\tau_{a}, \tau_{\varphi}>0$.

The quadratic term in Eq. (7) appears due to the assumption that the noise in (2) is a zero-mean white Gaussian. The sparsity assumption implies that phase and amplitude can be well approximated by spectrum vectors $\boldsymbol{\theta}_{a}$ and $\boldsymbol{\theta}_{\varphi}$ with a small number of nonzeros. Thus, we wish to minimize the norms $\left\|\boldsymbol{\theta}_{a}\right\|_{p}$ and $\left\|\boldsymbol{\theta}_{\varphi}\right\|_{p}$ in (7). 
Using (7) as the objective function and Eqs. (5)-(6) as the constraints linking the spectral and signal variables, the wave field reconstruction can be formalized as the constrained minimization.

\section{B. Multi-objective optimization: decoupling of inversion and filtering}

In this paper instead of a single-objective minimization outlined in the previous subsection, we propose an algorithm based on multi-objective optimization. This technique is very different from the conventional settings with a single objective function. The motivations of this approach are as follows:

(A) The algorithm produces a better imaging and better accuracy than those of the algorithms using the fidelity and regularization terms together in single-objective optimization.

(B) The algorithm is simple in implementation because the inversion and the filtering are decoupled. An efficient procedure based on Fast Fourier Transform (FFT) is developed for the inversion step, and an efficient algorithm based of hard- and soft- thresholding is developed for the phase and amplitude filtering.

The proposed algorithm is based on a joint solution of two constrained optimization problem:

$$
\begin{gathered}
\hat{\mathbf{u}}_{0}=\arg \min _{\mathbf{u}_{0}} \frac{1}{\mu}\left\|\mathbf{y}-\mathbf{A}_{z} \cdot \mathbf{u}_{0}\right\|_{2}^{2}, \\
\text { subject to }\left\|\mathbf{u}_{0}-\boldsymbol{\Psi}_{a} \hat{\boldsymbol{\theta}}_{a} \circ \exp \left(j \boldsymbol{\Psi}_{\varphi} \hat{\boldsymbol{\theta}}_{\varphi}\right)\right\|_{2}^{2} \leq \varepsilon_{1}, \\
\left(\hat{\boldsymbol{\theta}}_{a}, \hat{\boldsymbol{\theta}}_{\varphi}\right)=\arg \min _{\boldsymbol{\theta}_{a}, \boldsymbol{\theta}_{\varphi}} \tau_{a} \cdot\left\|\boldsymbol{\theta}_{a}\right\|_{l_{p}}+\tau_{\varphi} \cdot\left\|\boldsymbol{\theta}_{\varphi}\right\|_{l_{p}}, \\
\text { subject to }\left\|\boldsymbol{\theta}_{a}-\boldsymbol{\Phi}_{a} \cdot \operatorname{abs}\left(\hat{\mathbf{u}}_{0}\right)\right\| \leq \varepsilon_{2},\left\|\boldsymbol{\theta}_{\varphi}-\boldsymbol{\Phi}_{\varphi} \cdot \operatorname{angle}\left(\hat{\mathbf{u}}_{0}\right)\right\|_{2}^{2} \leq \varepsilon_{3} .
\end{gathered}
$$

In these formulas $\|\cdot\|_{2}^{2}$ stands for the Euclidean norm, and $\varepsilon_{1}, \varepsilon_{2}, \varepsilon_{3}>0$ are parameters. 
The inequalities in (9) and (11) relax the equalities (5)-(6) linking amplitude and phase of the wave field with the corresponding spectrum variables. For $\varepsilon_{1}, \varepsilon_{2}, \varepsilon_{3} \rightarrow 0$ these inequality constraints give the equalities (5)-(6).

In the used approach there is no a single criterion function. Instead we are looking for a fixed-point $\left(\hat{\mathbf{u}}_{0}, \hat{\boldsymbol{\theta}}_{a}, \hat{\boldsymbol{\theta}}_{\varphi}\right)$ defined as a solution of two optimization problem. In (8) the observations $\mathbf{y}$ are fitted by selecting an optimal $\mathbf{u}_{0}$ provided the given spectra $\left(\hat{\boldsymbol{\theta}}_{a}, \hat{\boldsymbol{\theta}}_{\varphi}\right)$, and in (10) the sparsest spectral model, minimizing $\tau_{a} \cdot|| \boldsymbol{\theta}_{a}\left\|_{l_{p}}+\tau_{\varphi} \cdot\right\| \boldsymbol{\theta}_{\varphi}||_{l_{p}}$, is selected provided the given $\hat{\mathbf{u}}_{0}$. The fixed-point $\left(\hat{\mathbf{u}}_{0}, \hat{\boldsymbol{\theta}}_{a}, \hat{\boldsymbol{\theta}}_{\varphi}\right)$ defines a balance between these two different and, in general, alternative goals.

Note also, that minimization of (8) on $\mathbf{u}_{0}$ performs the inversion of the forward propagation operator provided the given spectra $\hat{\boldsymbol{\theta}}_{a}, \hat{\boldsymbol{\theta}}_{\varphi}$, and minimization on $\boldsymbol{\theta}_{a}, \boldsymbol{\theta}_{\varphi}$ in (10) performs the filtering of amplitude and phase provided the given wave field $\hat{\mathbf{u}}_{0}$. Thus, as a solution of (8)-(11), the inversion and filtering are decoupled operations.

The constrained minimizations in (8)-(9) and (10)-(11) can be replaced by unconstrained ones using quadratic penalties instead of the inequalities in (9) and (11). In order to do it we introduce the corresponding two criterion functions

$$
\begin{aligned}
& \mathcal{L}_{1}\left(\mathbf{u}_{0}, \boldsymbol{\theta}_{a}, \boldsymbol{\theta}_{\varphi}\right)=\frac{1}{\mu}\left\|\mathbf{y}-\mathbf{A}_{z} \cdot \mathbf{u}_{0}\right\|_{2}^{2}+ \\
& \frac{1}{\gamma_{0}}\left\|\mathbf{u}_{0}-\boldsymbol{\Psi}_{a} \boldsymbol{\theta}_{a} \circ \exp \left(j \boldsymbol{\Psi}_{\varphi} \boldsymbol{\theta}_{\varphi}\right)\right\|^{2}
\end{aligned}
$$

and

$$
\begin{aligned}
& \mathcal{L}_{2}\left(\mathbf{u}_{0}, \boldsymbol{\theta}_{a}, \boldsymbol{\theta}_{\varphi}\right)=\tau_{a} \cdot\left\|\boldsymbol{\theta}_{a}\right\|_{l_{p}}+\tau_{\varphi} \cdot\left\|\boldsymbol{\theta}_{\varphi}\right\|_{l_{p}}+ \\
& \frac{1}{2 \gamma_{a}}\left\|\boldsymbol{\theta}_{a}-\boldsymbol{\Phi}_{a} \cdot \bmod \left(\mathbf{u}_{0}\right)\right\|^{2}+\frac{1}{2 \gamma_{\varphi}}\left\|\boldsymbol{\theta}_{\varphi}-\boldsymbol{\Phi}_{\varphi} \cdot \operatorname{angle}\left(\mathbf{u}_{0}\right)\right\|^{2}
\end{aligned}
$$

for the problems (8)-(9) and (10)-(11), respectively. 
Then the constrained optimizations in (8)-(11) are replaced by the following unconstrained ones

$$
\begin{aligned}
\hat{\mathbf{u}}_{0} & =\arg \min _{\mathbf{u}_{0}} \mathcal{L}_{1}\left(\mathbf{u}_{0}, \hat{\boldsymbol{\theta}}_{a}, \hat{\boldsymbol{\theta}}_{\varphi}\right) \\
\left(\hat{\boldsymbol{\theta}}_{a}, \hat{\boldsymbol{\theta}}_{\varphi}\right) & =\arg \min _{\boldsymbol{\theta}_{a}, \boldsymbol{\theta}_{\varphi}} \mathcal{L}_{2}\left(\hat{\mathbf{u}}_{0}, \boldsymbol{\theta}_{a}, \boldsymbol{\theta}_{\varphi}\right) .
\end{aligned}
$$

A replacement of the constraints by the penalties with positive parameters $\left(\gamma_{0}, \gamma_{a}\right.$ and $\left.\gamma_{\varphi}\right)$ is one of the standard tools to deal with the constrained optimization [26]. The problems (8)-(11) and (16) are equivalent provided a proper selection of the parameters $\gamma_{0}, \gamma_{a}, \gamma_{\varphi}$.

The proposed algorithm solving the problem (16) has the following iterative form:

$$
\begin{array}{r}
\mathbf{u}_{0}^{t+1}=\arg \min _{\mathbf{u}_{0}} \mathcal{L}_{1}\left(\mathbf{u}_{0}, \boldsymbol{\theta}_{a}{ }^{t}, \boldsymbol{\theta}_{\varphi}{ }^{t}\right), \\
\left(\boldsymbol{\theta}_{a}{ }^{t+1}, \boldsymbol{\theta}_{\varphi}{ }^{t+1}\right)=\arg \min _{\boldsymbol{\theta}_{a}, \boldsymbol{\theta}_{\varphi}} \mathcal{L}_{2}\left(\mathbf{u}_{0}^{t+1}, \boldsymbol{\theta}_{a}, \boldsymbol{\theta}_{\varphi}\right), \\
t=0,1, \ldots
\end{array}
$$

The algorithm (17)-(18) is a generalization of the decoupled inverse imaging developed in the recent papers $[6,7]$. It is demonstrated in these papers that the decoupling applied together with the BM3D-frame image modeling results in the imaging which is advanced with respect to that can be achieved using a single-objective optimization. Motivated by these promising results we modified this technique for the wave field reconstruction.

Concerning the theory behind of the proposed algorithm we wish to note that a convergence in-small (in a small neighborhood of the fixed-point $\left(\hat{\mathbf{u}}_{0}, \hat{\boldsymbol{\theta}}_{a}, \hat{\boldsymbol{\theta}}_{\varphi}\right)$ can be proved for (17)-(18) using the technique developed in [7].

The algorithm (17)-(18) as well as the constrained minimization in (8)-(11) allow an interesting interpretation in terms of the game theory (e.g. [27,28]). Minimization of $\mathcal{L}_{1}\left(\mathbf{u}_{0}, \boldsymbol{\theta}_{a}, \boldsymbol{\theta}_{\varphi}\right)$ 
on $\mathbf{u}_{0}$ and $\mathcal{L}_{2}\left(\mathbf{u}_{0}, \boldsymbol{\theta}_{a}, \boldsymbol{\theta}_{\varphi}\right)$ on $\left(\boldsymbol{\theta}_{a}, \boldsymbol{\theta}_{\varphi}\right)$ can be treated as a game of two players identified, respectively, with two groups of variables $\mathbf{u}_{0}$ and $\boldsymbol{\theta}_{a}, \boldsymbol{\theta}_{\varphi}$.

It can be seen that an interaction between these players is noncooperative since the minimization of $\mathcal{L}_{1}\left(\mathbf{u}_{0}, \boldsymbol{\theta}_{a}, \boldsymbol{\theta}_{\varphi}\right)$ on $\mathbf{u}_{0}$, in general, results in increasing of $\mathcal{L}_{2}\left(\mathbf{u}_{0}, \boldsymbol{\theta}_{a}, \boldsymbol{\theta}_{\varphi}\right)$, and the minimization of $\mathcal{L}_{2}\left(\mathbf{u}_{0}, \boldsymbol{\theta}_{a}, \boldsymbol{\theta}_{\varphi}\right)$ on $\left(\boldsymbol{\theta}_{a}, \boldsymbol{\theta}_{\varphi}\right)$ can increase $\mathcal{L}_{1}\left(\mathbf{u}_{0}, \boldsymbol{\theta}_{a}, \boldsymbol{\theta}_{\varphi}\right)$.

The iterative algorithm (17)-(18) models a selfish behavior of the variables (players) each minimizing only its own objective function. In the game theory the fixed-point (16) is known as the generalized Nash equilibrium.

\section{Algorithm development}

\section{A. Optimization}

Minimization on $\mathbf{u}_{0}$ and $\boldsymbol{\theta}_{a}, \boldsymbol{\theta}_{\varphi}$ respectively for (17) and (18) results in solutions which can be given in analytical forms. The corresponding derivations are presented in Appendix. Using these results we arrive at the following procedures:

(1) Minimization on $\boldsymbol{\theta}_{a}$ and $\boldsymbol{\theta}_{\varphi}$ in (18) gives

$$
\begin{aligned}
\boldsymbol{\theta}_{a} & =\mathfrak{T h}_{\tau_{a} \gamma_{a}}\left\{\Phi_{a} \cdot \operatorname{abs}\left(\mathbf{u}_{0}\right)\right\} \\
\boldsymbol{\theta}_{\varphi} & =\mathfrak{T h}_{\tau_{\varphi} \gamma_{\varphi}}\left\{\Phi_{\varphi} \cdot \operatorname{angle}\left(\mathbf{u}_{0}\right)\right\}
\end{aligned}
$$

where $\mathfrak{T h}_{\tau}\{\cdot\}$ stands for the thresholding operator defined in Appendix, Eq. (32). The type of the operator (hard- or soft-thresholding) depends on the used norm $l_{0}$ or $l_{1}, \tau$ is the thresholding parameter; 
(2) Minimization on $\mathbf{u}_{0}$ in (17) gives

$$
\begin{aligned}
& \mathbf{u}_{0}=\left(\frac{1}{\mu} \mathbf{A}_{z}^{H} \mathbf{A}_{z}+\frac{1}{\gamma_{0}} \mathbf{I}_{n \times n}\right)^{-1}\left(\frac{1}{\mu} \mathbf{A}_{z}^{H} \mathbf{y}+\frac{1}{\gamma_{0}} \mathbf{v}_{0}\right), \\
& \mathbf{v}_{0}=\boldsymbol{\Psi}_{a} \boldsymbol{\theta}_{a} \circ \exp \left(j \boldsymbol{\Psi}_{\varphi} \boldsymbol{\theta}_{\varphi}\right)
\end{aligned}
$$

as it shown in Appendix, Eq. (28).

The analytical forms of the solutions (19) and (20) is an important advantage of the decoupled approach, where the optimization variables are split in two groups with minimization of $\mathcal{L}_{2}$ with respect to $\left(\boldsymbol{\theta}_{a}, \boldsymbol{\theta}_{\varphi}\right)$ and $\mathcal{L}_{1}$ with respect to $\mathbf{u}_{0}$. In this formulation the object distribution $\mathbf{u}_{0}$ depends on phase and amplitude only through $\mathbf{v}_{0}$. This variable separates the image domain $\mathbf{u}_{0}$ and the spectral domain $\boldsymbol{\theta}_{a}, \boldsymbol{\theta}_{\varphi}$ optimizations.

\section{B. Fourier domain calculations}

Eq. (1) is a matrix representation for the convolution of the object signal and the shiftinvariant kernel of the wave field propagation diffraction operator. Let us denote this kernel as $a_{z}$. Computation of the convolution using FFT requires that the computation window size (support of FFT) to be properly selected (e.g. [12]). It is shown in [14] that the computation window of the double size of the object distribution support is sufficient for the precise calculation of F-DDT. We use this result as well as the technique developed for F-DDT in [14] for

modeling the forward propagation (operator $\mathbf{A}_{z}$ ) in our experiments and for implementation of our algorithm. Note that this technique is developed for the Fresnel approximation of the Rayleigh-Sommerfield diffraction integral.

Let us consider details of these calculations as they are implemented in the proposed algorithm. Assume that the support of the object distribution $u_{0}$ is a rectangular aperture 
of the size $N_{1} \times N_{2}$, and the sensor output $u_{z}$ has a support of the same size. Introduce a virtual object aperture of the double size $2 N_{1} \times 2 N_{2}$.

Let $\tilde{u}_{0}$ be a double size extended object distribution defined on this virtual aperture in such a way that $u_{0}$ is located in the central part of the virtual aperture and zero padded outside $u_{0}$ up to the size of the virtual aperture.

Introduce also an auxiliary variable $v_{0}$ of the support $N_{1} \times N_{2}$ used in the algorithm. Similar to $\tilde{u}_{0}$ the variable $\tilde{v}_{0}$ has the central part equal to $v_{0}$, and it is zero padded to the double size $2 N_{1} \times 2 N_{2}$. In a similar way, $\tilde{y}$ has the central part equal to $y$, and it is zero padded to the double size $2 N_{1} \times 2 N_{2}$.

Introduce a virtual aperture for the sensor which is of the same double size $2 N_{1} \times 2 N_{2}$. Let $\tilde{u}_{z}$ be a wave field distribution defined for this double size virtual sensor as it is generated by the wave field propagated from the object plane. The central part of $\tilde{u}_{z}$ of the size $N_{1} \times N_{2}$ corresponds to the actual sensor with the wave field distribution $u_{z}=y$.

Let $\tilde{a}_{z}$ denote the kernel of the wave field propagation operator of the size equal to size of the virtual aperture $2 N_{1} \times 2 N_{2}$.

FFTs for the introduced double size variables $\tilde{u}_{0}, \tilde{u}_{z}, \tilde{v}_{0}, \tilde{a}_{z}$ are defined as $\tilde{U}_{0}(f)=$ $\mathcal{F F} \mathcal{T}\left(\tilde{u}_{0}\right), \tilde{U}_{z}(f)=\mathcal{F} \mathcal{F} \mathcal{T}\left(\tilde{u}_{z}\right), \tilde{V}_{0}(f)=\mathcal{F F} \mathcal{T}\left(\tilde{v}_{0}\right), \tilde{A}_{z}(f)=\mathcal{F} \mathcal{F} \mathcal{T}\left(\tilde{a}_{z}\right)$, where $f$ is the $2 D$ integer FFT frequency. We use the shifted FFT, where the zero-frequency component is shifted to center of spectrum. Then $u_{z}$ corresponding to the vector $\mathbf{u}_{z}$ in Eq. (1) is calculated according to the following formulas [14]:

$$
\tilde{U}_{z}(f)=\tilde{A}_{z}(f) \tilde{U}_{0}(f), \tilde{u}_{z}=\mathcal{F} \mathcal{F} \mathcal{T}^{-1}\left(\tilde{U}_{z}(f)\right), u_{z}=\tilde{u}_{z}(\text { center })
$$

where $\tilde{u}_{z}($ center $)$ stands for the central part of $\tilde{u}_{z}$ of the size $N_{1} \times N_{2}$. 
The links between the matrix and FFT domain operations for the double size variables give the precise FFT analog of the matrix formula (20)

$$
\begin{aligned}
& \tilde{U}_{0}(f)=\left(\frac{1}{\mu} \tilde{A}_{z}^{*}(f) \tilde{Y}(f)+\frac{1}{\gamma_{0}} \tilde{V}_{0}\right) /\left(\frac{1}{\mu}\left|\tilde{A}_{z}(f)\right|^{2}+\frac{1}{\gamma_{0}}\right), \\
& \tilde{u}_{0}=\mathcal{F} \mathcal{F} \mathcal{T}^{-1}\left(\tilde{U}_{0}(f)\right), u_{0}=\tilde{u}_{0}(\text { center }),
\end{aligned}
$$

where $(*)$ stand for complex conjugate variables.

\section{C. Proposed algorithm}

For initialization of the algorithm (17)-(18) we use the regularized inverse (RI) solution $u_{0}^{R I}$ calculated as

$$
\begin{aligned}
& \tilde{U}^{R I}(f)=\frac{1}{\mu} \tilde{A}^{*}(f) \tilde{Y}(f) /\left(\frac{1}{\mu}|\tilde{A}(f)|^{2}+\frac{1}{\gamma}\right), \\
& \tilde{u}_{0}^{R I}=\mathcal{F} \mathcal{F} \mathcal{T}^{-1}\left(\tilde{U}^{R I}(f)\right), u_{0}^{R I}=\tilde{u}_{0}^{R I}(\text { center }),
\end{aligned}
$$

where $\gamma>0$ is a regularization parameter.

Using Eqs. (19), (22), (23) and FFT calculations described in Subsection 5.B, the algorithm 
(17)-(18) can be presented in the following form.

\section{SPAR algorithm}

Input : $y, \tilde{A}(f), u_{0}^{R I}$

Using $u_{0}^{R I}$ construct transforms $\boldsymbol{\Phi}$ and $\boldsymbol{\Psi}$

Set $t=0, u_{0}^{0}=u_{0}^{R I}, u_{z}^{0}=y, v_{0}^{0}=y$,

Repeat until convergence :

1. $\boldsymbol{\theta}_{a}^{t}=\mathfrak{T h}_{\tau_{a} \gamma_{a}}\left\{\boldsymbol{\Phi}_{a} \cdot \operatorname{abs}\left(\mathbf{u}_{0}^{t}\right)\right\}$

2. $\boldsymbol{\theta}_{\varphi}^{t}=\mathfrak{T h}_{\tau_{\varphi \gamma_{\varphi}}}\left\{\boldsymbol{\Phi}_{\varphi} \cdot \operatorname{angle}\left(\mathbf{u}_{0}^{t}\right)\right\}$

3. $\mathbf{v}_{0}^{t}=\boldsymbol{\Psi}_{a} \boldsymbol{\theta}_{a}^{t} \circ \exp \left(j \Psi_{\varphi} \boldsymbol{\theta}_{\varphi}^{t}\right)$

4. $\tilde{U}_{0}^{t+1 / 2}(f)=\left(\frac{1}{\mu} \tilde{A}^{*}(f) \tilde{U}_{z}^{t}(f)+\frac{1}{\gamma_{0}} \tilde{V}_{0}^{t}\right) /\left(\frac{1}{\mu}|\tilde{A}(f)|^{2}+\frac{1}{\gamma_{0}}\right)$,

5. $\tilde{u}_{0}^{t+1}=\tilde{u}_{0}^{t+1 / 2}($ center $), \tilde{U}_{0}^{t+1}(f)=\mathcal{F} \mathcal{F} \mathcal{T}\left(\tilde{u}_{0}^{t+1}\right)$

6. $\tilde{U}_{z}^{t+1}(f)=\tilde{A}(f) \cdot \tilde{U}_{0}^{t+1}(f), \tilde{u}_{z}^{t}=\mathcal{F} \mathcal{F} \mathcal{T}^{-1}\left(\tilde{U}_{z}^{t+1}(f)\right)$

7. $\tilde{u}_{z}^{t+1}($ center $)=y, \tilde{U}_{z}^{t+1}(f)=\mathcal{F} \mathcal{F} \mathcal{T}\left(\tilde{u}_{z}^{t+1}\right)$,

$t=t+1$.

The upper index ' $t$ ' stands for the iteration number. Bold $\mathbf{u}_{0}^{t}$ and $\mathbf{v}_{0}^{t}$ denote vectors, while $u_{0}^{t}$ and $v_{0}^{t}$ stand for the corresponding $N_{1} \times N_{2}$ images reshaped from the vectors $\mathbf{u}_{0}^{t}$ and $\mathbf{v}_{0}^{t}$.

The frames (transforms) $\boldsymbol{\Phi}$ and $\boldsymbol{\Psi}$ are constructed at the initial step in line (26) for the initial estimate $u_{0}^{R I}$ and fixed through further iterations.

In Steps 1 and $2, \mathfrak{T}\{\cdot\}$ means the thresholding operators with the parameters $\tau_{a} \gamma_{a}$ and $\tau_{\varphi} \gamma_{\varphi}$. These elementwise operators are applied to the vectors $\boldsymbol{\Phi}_{a} \cdot \operatorname{abs}\left(\mathbf{u}_{0}^{t}\right)$ and $\boldsymbol{\Phi}_{\varphi} \cdot \operatorname{angle}\left(\mathbf{u}_{0}^{t}\right)$.

The estimates of amplitude $\boldsymbol{\theta}_{a}^{t}$ and phase $\boldsymbol{\theta}_{\varphi}^{t}$ spectra are used for calculation of $\mathbf{v}_{0}^{t}$ in Step 
3.

At Step 4, a preliminary estimate $\tilde{U}_{0}^{t+1 / 2}$ of the double size object wave field is updated. At Step 5, this estimate is zero-padded in the image domain, and the final double size estimate is returned in the FFT domain as $\tilde{U}_{0}^{t+1}(f)$.

The double size estimate $\tilde{U}_{z}^{t}(f)$ at the sensor plane is calculated at Step 6 . The central part of this estimate is replaced by the given observations at Step 7. Note, that this double size estimate $\tilde{u}_{z}^{t+1}$ defines the essential advantage of the double size virtual sensor utilizing the estimates outside the actual sensor [14].

We name the proposed algorithm Sparse Phase Amplitude Reconstruction (SPAR).

If the sparse representations and thresholdings (Steps 1,2) are dropped and $\tilde{V}_{0} \equiv 0$ in Step 4, the proposed algorithm becomes identical to the iterative regularized inverse F-DDT algorithm from [14]. The spectrum-transform representations of phase and amplitude and their filtering in SPAR differ these algorithms.

\section{Numerical experiments}

We consider the object wave fields with the amplitude and/or phase modulation in the form $u_{0}[k]=\left|u_{0}[k]\right| \cdot \exp \left(j \cdot \varphi_{0}[k]\right)$. For the amplitude modulation $\varphi_{0}[k] \equiv 0, \operatorname{abs}\left(u_{0}[k]\right)=w[k]+.2$. For the phase modulation abs $\left(u_{0}[k]\right) \equiv 1, \varphi_{0}[k]=\pi(w[k]-1 / 2) / 5$. Here $w[k], 0 \leq w[k] \leq 1$, is a spatially varying test-image. The results are shown for two square $(256 \times 256)$ test-images: binary chessboard and gray-scale cameraman.

Pixelated (discrete) models for the object and sensor planes have pixels $\Delta \times \Delta$ with $\Delta=6.7 \mu \mathrm{m}$ and $100 \%$ fill factors. The wavelength $\lambda=532 \mathrm{~nm}$ corresponds to a green laser. "In-focus" distance is calculated as $z_{f}=N_{1} \cdot \Delta^{2} / \lambda, N_{1}=256[2]$. 
The double size F-DDT is used for the forward propagation kernel $a_{z}$ in the observation modeling and in the algorithm. Recall that this F-DDT technique enables the exact wave field propagation for pixelated sensor and object distributions [14].

Comparison of reconstructions obtained using hard- and soft-thresholding $\left(l_{0}\right.$-or $l_{1}$-norms for penalization, respectively) is in favor of the formers. In what follows we present results obtained using the hard-shresholding ( $l_{0}$-norm) only.

The parameters of the algorithm are fixed as follows: $\mu=1, \tau_{a} \gamma_{a}=0.02, \tau_{\varphi} \gamma_{\varphi}=0.02$, $\gamma=100$ For noiseless data $\gamma_{0}=1$ and for noisy data $\gamma_{0}=1 / 5$. These basic values of the algorithm's parameters are found experimentally as enabling good performance of the algorithm in all our experiments. Experimental results are shown for 200 iterations of the algorithm.

The computational complexity of the algorithm is characterized by the time required for calculations. For 200 iterations it takes about 150 sec. for the computer and the software used in the experiments: Intel Core 2Duo E8400 @ 3GHz, RAM 4GB, Windows Xp SP3, Matlab 7.9.0 (R2009b).

For comparison with respect to the conventional techniques we show the phase and amplitude reconstructions obtained using the ASD backpropagation algorithm.

The presented figures for experimental results are organized in the following way. The first line are images for the amplitude and phase reconstructions. The second line shows crosssections of these images. The first column in the figures is for the amplitude reconstructions, and the second column is for the phase reconstructions.

Following the principle of the reproducible research [29] we make our MATLAB programs for the demo version of SPAR publicly available for testing: 
http://www.cs.tut.fi/ lasip/DDT/.

\section{A. Noiseless data}

The results obtained by the ASD algorithm are shown in Fig.1. The phase modulation object is considered with the chessboard test-image for the phase. Both the amplitude and the phase reconstructions are seriously damaged by multiple artifacts (wiggles, ringing, waves, etc.) clearly seen in the cross-sections. This sort of artifacts are typical for all kinds of the conventional numerical techniques.

Results in Fig.2 are shown for the same data as in Fig.1 but obtained using the proposed SPAR algorithm. Visually, the reconstruction is perfect for both the amplitude and phase.

In the cross-sections a difference between the reconstructions and the true values of phase and amplitude is not seen.

As a more difficult for reconstruction scenario we consider a complex-valued object where both the amplitude and phase are spatially varying. For the amplitude modulation we use the binary chessboard image. For the phase modulation we use the gray scale cameraman. The results in Fig.3 are obtained by the ASD algorithm. The visual quality of the reconstruction is very poor: typical artifacts are clearly seen as well as the chessboard squares (from amplitude modulation) in the phase reconstruction image. Contrary to it the SPAR algorithm (Fig.4) resolves the phase and the amplitude information perfectly with clear and accurate imaging for the both variables. In the cross-sections thick ('red' in color) and thin ('blue' in color) lines show the true signal and reconstruction, respectively.

The accuracy of the reconstruction is characterized by the root-mean-squared-error (RMSE) criterion calculated independently for phase and amplitude. The corresponding 
values of RMSE are shown in the figures.

\section{B. Noisy data}

For the noisy data we use $\sigma=.02$. The results for the gray-scale phase modulation (test-image cameraman) are shown in Fig.5 and Fig.6 for the ASD and SPAR algorithms, respectively. The advantage of the SPAR algorithm is obvious visually and numerically. The cross-sections for the SPAR algorithm show that the high-accuracy reconstructions are obtained for both the invariant amplitude and the spatially varying phase. For the ASD algorithm the reconstructions are quite noisy, and the typical artifacts (wiggles and waves) are clearly seen.

\section{C. Comparative accuracy}

$R M S E$ versus the number of iterations are presented in Fig. 7 for the reconstructions shown in Fig. 2. This curves demonstrate the computational price of the higher accuracy reconstruction versus the number of iterations. Say, if we stop after 100 iterations we have $R M S E_{a}=0.0075, R M S E_{\varphi}=0.008$ versus $R M S E_{a}=0.0016, R M S E_{\varphi}=0.0016$ for 200 iterations. This accuracy improvement can be considered as quite essential. However, the accuracy after 100 iterations is already very high, and the algorithm can be stopped.

All the above results are obtained for the in-focus distance between the object and sensor planes.

RMSE values in Tables 1 and 2 demonstrate the comparative accuracy of the ASD and SPAR algorithms for the in-focus and out-of-focus experiments. The distance between the image and object planes is calculated as $z=d z_{f}$, where $z_{f}$ is the in-focus distance and $d=1,2,3,5$. The strong advantage of the SPAR algorithm is obvious in all experiments. 


\section{Conclusion}

Simulation experiments demonstrate a very good performance of the proposed algorithm. The artifacts typically appeared in the conventional techniques are nearly eliminated. The accuracy of reconstruction is good for both phase and amplitude of the wave field in the object plane. Three components of the proposed approach enable the advance performance of the algorithm. First, the image modeling and the regularization are separate for phase and amplitude. Second, the BM3D-frames provide rich and overcomplete adaptive dictionaries for sparse approximations of phase and amplitude. Third, instead of a single objective function typical for the conventional sparse imaging techniques we use a vector optimization with two objective functions. The algorithm searches for a fixed-point giving a balance between two quality measures defined by these objective functions. This vector minimization decouples the inversion and the filtering operations and results in the iterative algorithm simple in implementation and highly efficient.

The developed algorithm is applicable in various optical setups where the accuracy of complex-valued wave field reconstruction is of special interest.

\section{Acknowledgments}

This work was supported by the Academy of Finland: project no. 213462, 2006-2011 (Finnish Programme for Centres of Excellence in Research) and project no. 138207, 2011-2014. The authors want to thank the reviewers for their constructive comments and suggestions. 


\section{References}

1. J. W. Goodman, Introduction to Fourier Optics, 3rd ed. (Roberts \& Company, Englewood, 2005).

2. Th. Kreis, Handbook of Holographic Interferometry: Optical and Digital Methods (WileyVCH, 2005).

3. M. Elad, Sparse and Redundant Representations: From Theory to Applications in Signal and Image Processing (Springer, 2010).

4. J. L. Starck, F. Murtagh, J. Fadili, Sparse Image and Signal Processing: Wavelets, Curvelets, Morphological Diversity (Cambridge University Press, 2010).

5. A. Danielyan, V. Katkovnik, and K. Egiazarian, "Image deblurring by augmented Lagrangian with BM3D frame prior," in Proceedings of Workshop on Information Theoretic Methods in Science and Engineering (WITMSE), Tampere, Finland, August 16 $18,2010$.

6. V. Katkovnik, A. Danielyan, and K. Egiazarian, "Decoupled inverse and denoising for image deblurring: variational BM3D-frame technique," in Proceedings of IEEE International Conference on Image Processing (ICIP, 2011).

7. A. Danielyan, V. Katkovnik, and K. Egiazarian, "BM3D frames and variational image deblurring," IEEE Trans. Image Processing (2011, submitted), arXiv:1106.6180v1.

8. K. Dabov, A. Foi, V. Katkovnik, and Egiazarian, K., "Image denoising by sparse 3D transform-domain collaborative filtering," IEEE Trans. Image Processing, 16, 2080 $2095(2007)$.

9. V. Katkovnik, A. Foi, K. Egiazarian, and J. Astola, "From local kernel to nonlocal 
multiple-model image denoising," International Journal of Computer Vision, 86, 1-32 $(2010)$

10. P. Chatterjee and P. Milanfar, "Is denoising dead?", IEEE Trans. Image Processing vol. 19, no. 4, 895-911 (2010).

11. V. Katkovnik and J. Astola, "Beyond the diffraction limits: inverse numerical imaging based on transform-domain sparse modeling for phase and amplitude," in Proceedings of 10th Euro-American Workshop on Information Optics (WIO-2011), Benicassim (Spain), 19-24 June, 2011.

12. F. Shen and A. Wang, "Fast-Fourier-transform based numerical integration method for the Rayleigh-Sommerfeld diffraction formula," Appl. Opt. 45, 1102-1110 (2006).

13. V. Katkovnik, A. Migukin, and J. Astola, "Backward discrete wave field propagation modeling as an inverse problem: toward perfect reconstruction of wave field distributions," Appl. Opt. 48, 3407-3423 (2009).

14. V. Katkovnik, J. Astola, and K. Egiazarian, "Discrete diffraction transform for propagation, reconstruction, and design of wavefield distributions, " Appl. Opt. 47, 3481-3493 (2008).

15. M. Bertero and P. Boccacci. Introduction to inverse problems in imaging. (IOP Publishing Ltd, 1998).

16. E. J. Candes, J. Romberg, and T. Tao, "Robust uncertainty principles: exact signal reconstruction from highly incomplete frequency information," IEEE Trans. Inf. Theory 52, 489-509 (2006).

17. E. J. Candes and T. Tao, "Near-optimal signal recovery from random projections: uni- 
versal encoding strategies" IEEE Trans. Inf. Theory 52, 5406-5425 (2006).

18. D. L. Donoho, "Compressed sensing," IEEE Trans. Inf. Theory 52, 1289-1306 (2006).

19. S. Gazit, A. Szameit, Y. C. Eldar, M. Segev, "Super-resolution and reconstruction of sparse sub-wavelength images, " Optics Express 17, 23920-23946 (2009).

20. D. J. Brady, K. Choi, D. L. Marks, R. Horisaki, and S. Lim, "Compressive holography," Optics Express, 17, 13040-13049 (2009).

21. C. F. Cull, D. A. Wikner, J. N. Mait, M. Mattheiss, and D. J. Brady, "Millimeter-wave compressive holography," Applied Optics, 49, 67-82. (2010).

22. K. Choi, R. Horisaki, J. Hahn, S. Lim, D.L.Marks, T.J. Schulz, D.J. Brady, "Compressive holography of diffuse objects," Applied Optics 49, H1-H10 (2010).

23. Y. Rivenson, A. Stern, B. Javidi, "Compressive Fresnel holography," Journal of Display Technology 6, 506-509 (2010).

24. Z. Xu and E. Y. Lam, "Image reconstruction using spectroscopic and hyperspectral information for compressive terahertz imaging," J. Opt. Soc. Am. A 27, 1638-1646 (2010).

25. D. Han, K. Kornelson, D. Larson, E. Weber, Frames for Undergraduates (Student Mathematical Library, AMS, 2007).

26. D. P. Bertsekas, Nonlinear Programming (Second ed., Cambridge, MA.: Athena Scientific, 1999).

27. M. J. Osborne, An Introduction to Game Theory (Oxford University Press, Inc., 2003).

28. F. Facchinei and Kanzow, C., "Generalized Nash Equilibrium Problems," Annals OR, pp. 177-211, 2010.

29. J. B. Buckheit and D. L. Donoho, "WaveLab and reproducible research," 
Dept. of Statistics, Stanford University, Tech. Rep. 474 (1995), http://www-

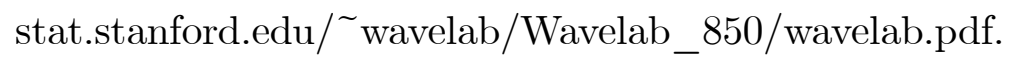


Appendix: Derivation of the algorithm

\section{Optimization of $\mathcal{L}_{1}$}

We use the minimum condition for (17) in the form $\partial \mathcal{L}_{1} / \partial \mathbf{u}_{0}^{*}=0$. It gives the equation linear with respect to $\mathbf{u}_{0}$

$$
\frac{1}{\mu} \mathbf{A}_{z}^{H}\left(\mathbf{A}_{z} \mathbf{u}_{0}-\mathbf{y}\right)+\frac{1}{\gamma_{0}}\left(\mathbf{u}_{0}-\mathbf{v}_{0}\right)=0
$$

and the solution in the form

$$
\begin{aligned}
& \hat{\mathbf{u}}_{0}=\left(\frac{1}{\mu} \mathbf{A}_{z}^{H} \mathbf{A}_{z}+\frac{1}{\gamma_{0}} \mathbf{I}_{n \times n}\right)^{-1} \times \\
& \times\left[\frac{1}{\mu} \mathbf{A}_{z}^{H} \mathbf{y}+\frac{1}{\gamma_{0}} \mathbf{v}_{0}\right] .
\end{aligned}
$$

\section{Optimization of $\mathcal{L}_{2}$}

Let us consider the optimization problem

$$
\min _{\boldsymbol{\theta}} \tau \cdot\|\boldsymbol{\theta}\|_{p}+\frac{1}{2}\|\boldsymbol{\theta}-\mathbf{B}\|_{2}^{2}
$$

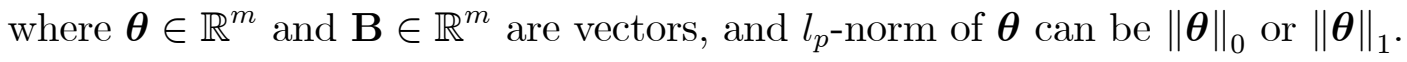

Due to the additive nature of the used norm the problem (29) can be solved independently for each component $\theta_{i}$ of the vector $\boldsymbol{\theta}$ :

$$
\theta_{i}=\arg \min _{\boldsymbol{\theta}_{i}} \tau \cdot\left\|\theta_{i}\right\|_{p}+\frac{1}{2}\left(\theta_{i}-\mathbf{B}_{i}\right)^{2}
$$

There is an analytical solution for (30) known as the hard-and soft-thresholding (or hardand soft-shrinkage) operators, respectively for the $l_{0}$ and $l_{1}$ norms.

Let us denote this operator as

$$
\boldsymbol{\theta}=\mathfrak{T h}_{\tau}\{\mathbf{B}\}
$$

where $\mathbf{B}$ is an input-vector and $\boldsymbol{\theta}$ is a solution-vector returned by the operator. 
For $l_{0}$ and $l_{1}$ this element-wise operator is specified as (e.g. [3])

$$
\begin{aligned}
& \boldsymbol{\theta}=\mathfrak{T h}_{\tau}\{\mathbf{B}\}= \\
& \left\{\begin{array}{c}
\mathfrak{T h}_{\tau}^{\text {soft }}\{\mathbf{B}\}=\operatorname{sign}(\mathbf{B}) \circ \max (|\mathbf{B}|-\tau, 0), \text { if } l_{p}=l_{1}, \\
\mathfrak{T h}_{\sqrt{2 \tau}}^{\text {hard }}\{\mathbf{B}\}=\mathbf{B} \circ 1(|\mathbf{B}| \geq \sqrt{2 \tau}), \text { if } l_{p}=l_{0},
\end{array}\right.
\end{aligned}
$$

where the indexes 'soft' and 'hard' indicate the type of the solution.

Note that the threshold parameters for the soft- and hard-thresholdings are different.

The solutions (19) are obtained from (32) and correspond to the minimization of $\tau_{a}$. $\left\|\boldsymbol{\theta}_{a}\right\|_{l_{p}}+\frac{1}{2 \gamma_{a}}\left\|\boldsymbol{\theta}_{a}-\Phi_{a} \cdot \bmod \left(\mathbf{u}_{0}\right)\right\|^{2}$ with respect to $\boldsymbol{\theta}_{a}$ and the minimization of $\tau_{\varphi} \cdot\left\|\boldsymbol{\theta}_{\varphi}\right\|_{l_{p}}+$ $\frac{1}{2 \gamma_{\varphi}}\left\|\boldsymbol{\theta}_{\varphi}-\Phi_{\varphi} \cdot \arg \left(\mathbf{u}_{0}\right)\right\|^{2}$ with respect to $\boldsymbol{\theta}_{\varphi}$. 


\section{List of Figure Captions}

Fig. 1 The phase modulation object with the binary phase (chessboard test-image), noiseless data. The reconstruction by the ASD algorithm. In the cross-sections thick ('red' in color) and thin ('blue' in color) lines show the true signal and the reconstructions, respectively. RMSE values for the phase and amplitude are shown at the top. The visual quality of the reconstruction is quite poor: wiggles, waves, etc. are clearly seen.

Fig.2 The phase modulation object with the binary phase (chessboard test-image), noiseless data. The reconstruction by the proposed SPAR algorithm. The visual quality of the reconstruction is perfect: wiggles, waves and other artifacts are completely wiped out. In the cross-sections the difference between the true signal and the reconstructions is not seen.

Fig.3 The amplitude modulation by the binary chessboard test-image and the phase modulation by the gray-scale spatially varying cameraman test-image, noiseless data. The reconstruction by the ASD algorithm. In the cross-sections thick ('red' in color) and thin ('blue' in color) lines show the true signal and the reconstructions, respectively. The visual quality of the reconstructions is very poor: artifacts as well as the chessboard squares (from amplitude modulation) are clearly seen in the phase reconstruction.

Fig.4 The amplitude modulation by the binary chessboard test-image and the phase modulation by the gray-scale cameraman test-image, noiseless data. The reconstruction by the proposed SPAR algorithm. The visual quality of the reconstructions is almost perfect. In the cross-sections a difference between the true signal and the reconstructions is slightly seen only in the phase reconstruction. 
Fig.5 The phase modulation by the gray-scale cameraman test-image, noisy data. The reconstruction by the ASD algorithm. In the cross-sections thick ('red' in color) and thin ('blue' in color) lines show the true signal and the reconstructions, respectively. The visual quality of the reconstructions is poor: artifacts and noise are well seen.

Fig.6 The phase modulation by the gray-scale cameraman test-image, noisy data. The reconstruction by the proposed SPAR algorithm. RMSE values for the phase and the amplitude are shown at the top. The visual quality of the reconstruction is quite good: wiggles, waves, other artifacts and noisy are nearly wiped out. The noise level in the reconstructions is quite low in comparison with what is achieved in Fig.5.

Fig.7 RMSE for the amplitude and phase reconstructions by the proposed SPAR algorithm versus the number of iterations. The phase modulation object with the binary phase (chessboard test-image), noiseless data. 


\section{List of Table Captions}

Table 1. RMSE values for the phase reconstruction. The phase modulation with the chessboard test-image for the phase. Comparison of the ASD and SPAR algorithms. Noiseless data.

Table 2. RMSE values for the phase reconstruction. The phase modulation with the cameraman test-image for the phase. Comparison of the ASD and SPAR algorithms. Noiseless data. 
Table 1. RMSE values for the phase reconstruction. The phase modulation with the chessboard test-image for the phase. Comparison of the ASD and SPAR algorithms. Noiseless data.

\begin{tabular}{||l||l||l||}
\hline \hline$d \backslash$ Algorithm & $A S D$ & $S P A R$ \\
\hline \hline 1 & 0.154 & 0.0016 \\
\hline \hline 2 & 0.185 & 0.0212 \\
\hline \hline 3 & 0.206 & 0.0288 \\
\hline \hline 5 & 0.238 & 0.0446 \\
\hline \hline
\end{tabular}

Table 2. RMSE values for the phase reconstruction. The phase modulation with the cameraman test-image for the phase. Comparison of the ASD and SPAR algorithms. Noiseless data.

\begin{tabular}{||l||l||l||}
\hline \hline$d \backslash$ Algorithm & $A S D$ & $S P A R$ \\
\hline \hline 1 & 0.151 & 0.011 \\
\hline \hline 2 & 0.182 & 0.0218 \\
\hline \hline 3 & 0.202 & 0.0311 \\
\hline \hline 5 & 0.231 & 0.0417 \\
\hline \hline
\end{tabular}


ASD, AMPL, RMSE = 0.0775
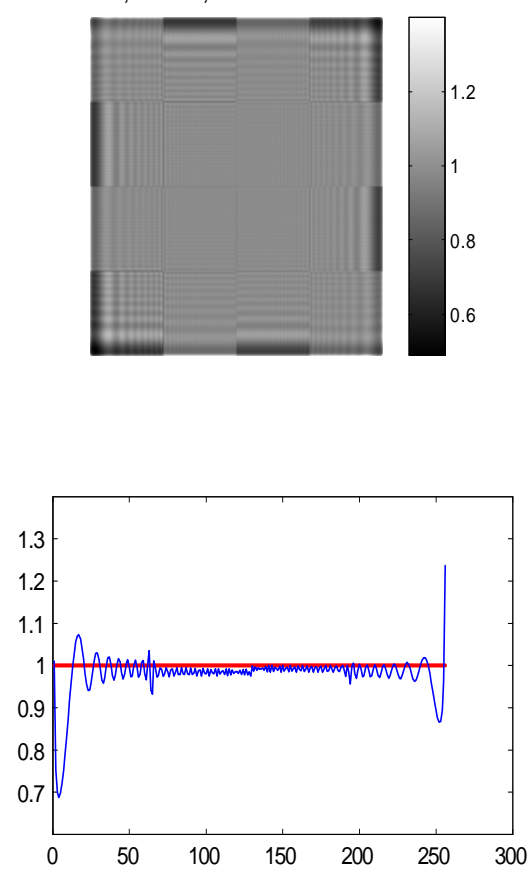

ASD, PHASE, RMSE $=0.154$
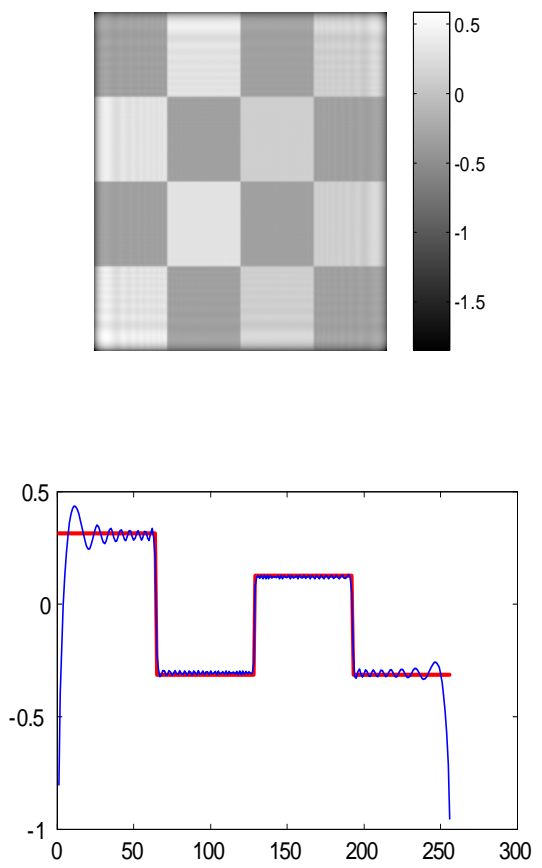

Fig. 1. The phase modulation object with the binary phase (chessboard testimage), noiseless data. The reconstruction by the ASD algorithm. In the crosssections thick ('red' in color) and thin ('blue' in color) lines show the true signal and the reconstructions, respectively. RMSE values for the phase and amplitude are shown at the top. The visual quality of the reconstruction is quite poor: wiggles, waves, etc. are clearly seen. 
SPAR, AMPL, RMSE $=0.0016$
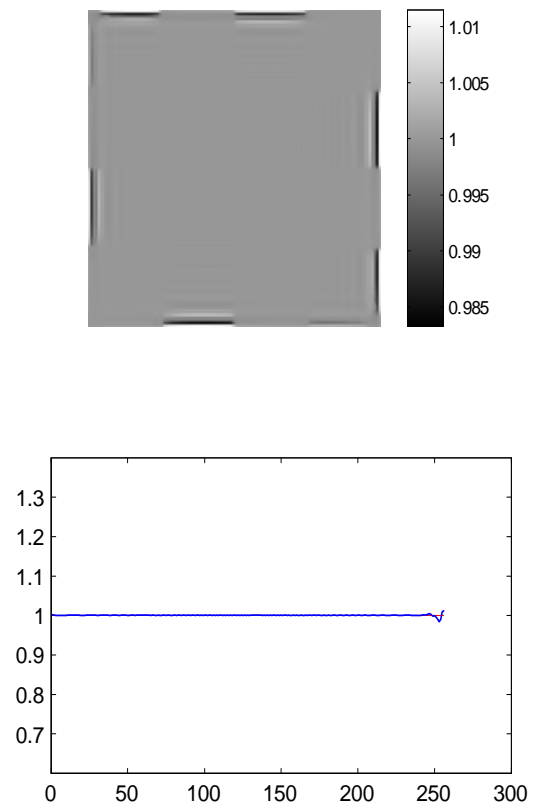

SPAR, PHASE, RMSE $=0.0016$
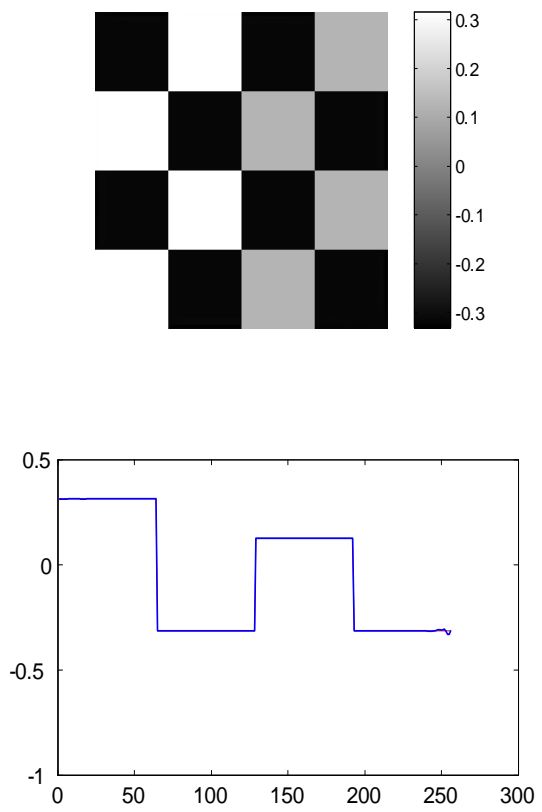

Fig. 2. The phase modulation object with the binary phase (chessboard testimage), noiseless data. The reconstruction by the proposed SPAR algorithm. The visual quality of the reconstruction is perfect: wiggles, waves and other artifacts are completely wiped out. In the cross-sections the difference between the true signal and the reconstructions is not seen. 

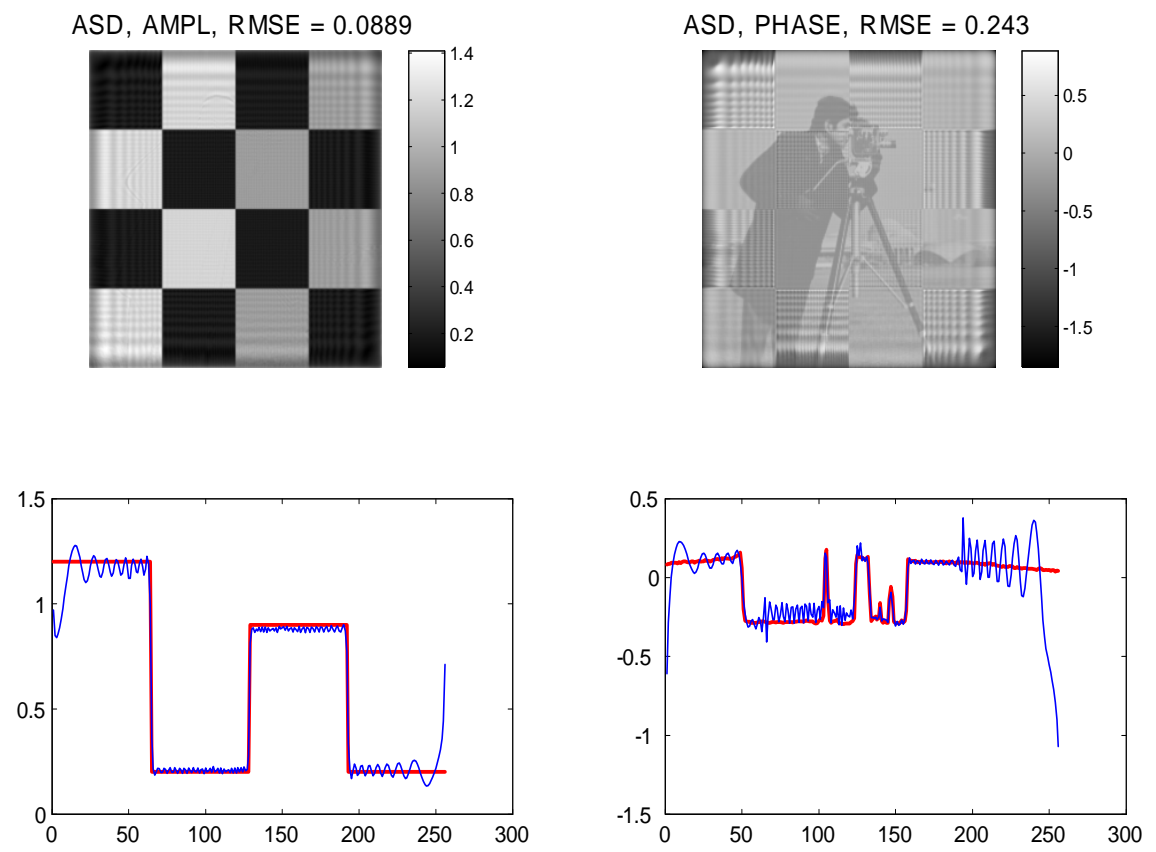

Fig. 3. The amplitude modulation by the binary chessboard test-image and the phase modulation by the gray-scale spatially varying cameraman test-image, noiseless data. The reconstruction by the ASD algorithm. In the cross-sections thick ('red' in color) and thin ('blue' in color) lines show the true signal and the reconstructions, respectively. The visual quality of the reconstructions is very poor: artifacts as well as the chessboard squares (from amplitude modulation) are clearly seen in the phase reconstruction. 

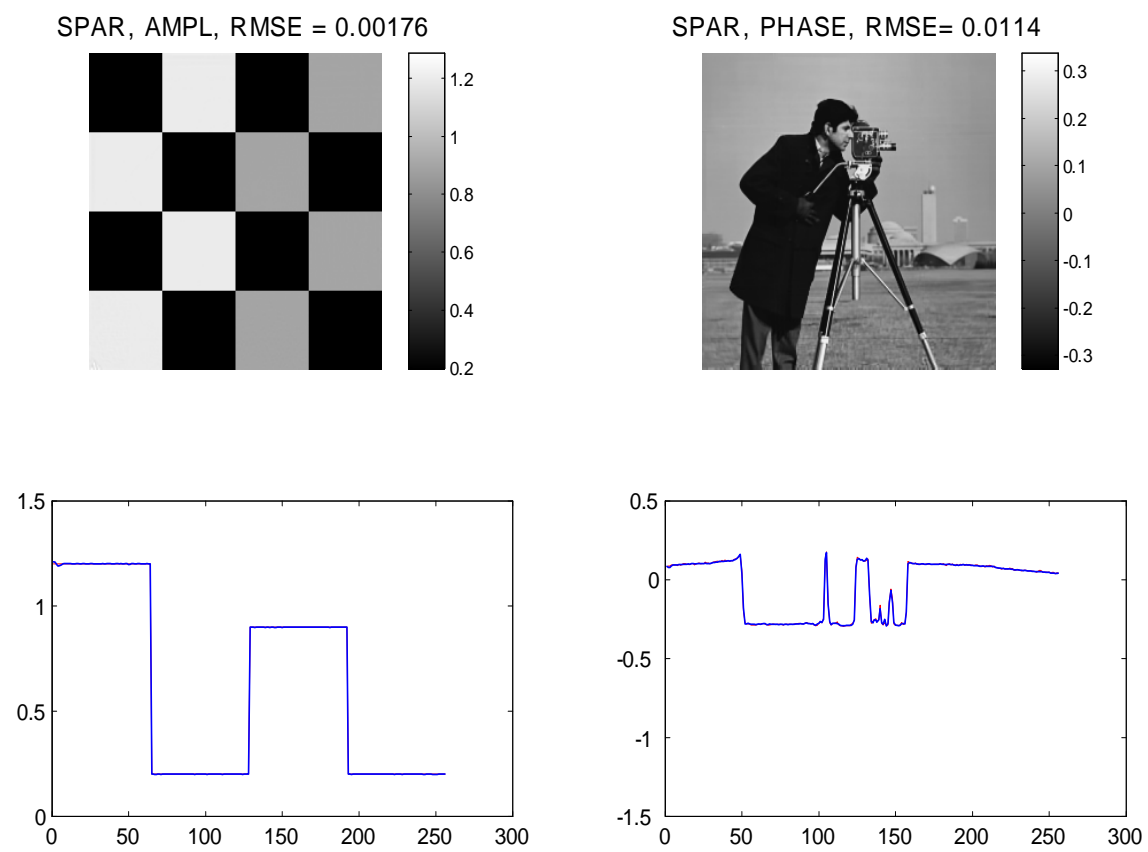

Fig. 4. The amplitude modulation by the binary chessboard test-image and the phase modulation by the gray-scale cameraman test-image, noiseless data. The reconstruction by the proposed SPAR algorithm. The visual quality of the reconstructions is almost perfect. In the cross-sections a difference between the true signal and the reconstructions is slightly seen only in the phase reconstruction. 
ASD, AMPL, RMSE $=0.072$
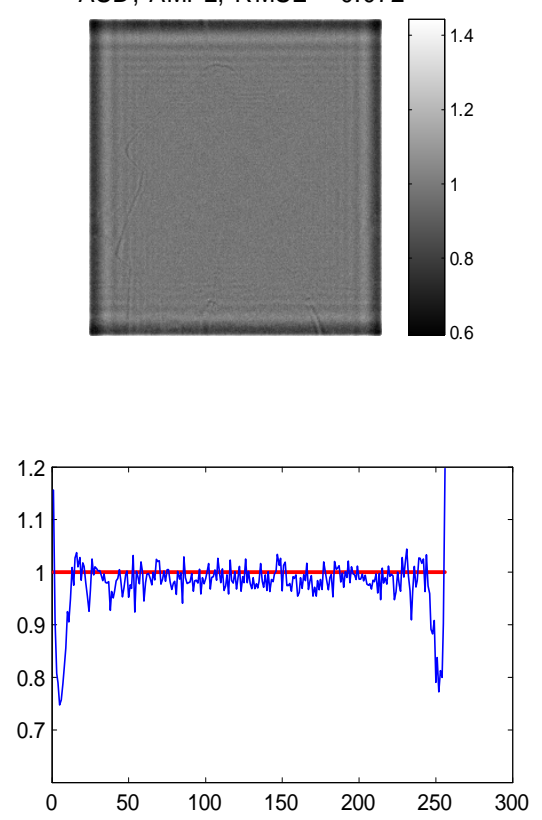

ASD, PHASE, RMSE $=0.153$
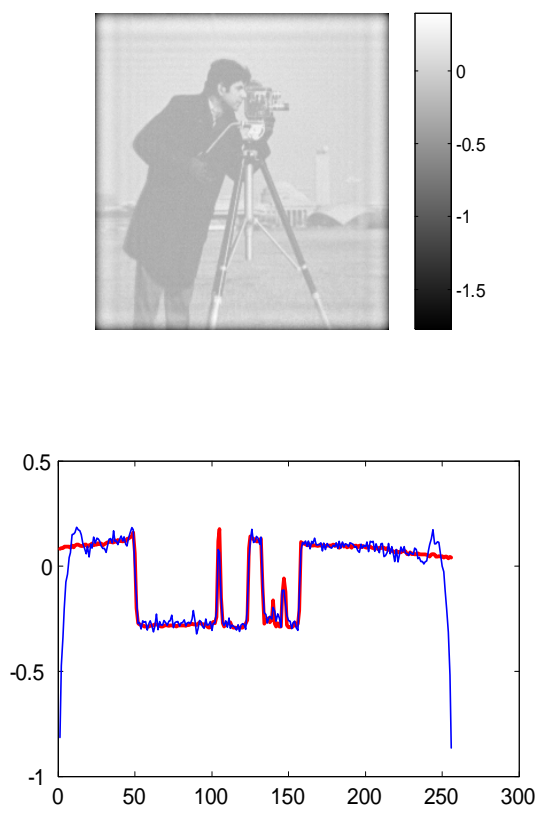

Fig. 5. The phase modulation by the gray-scale cameraman test-image, noisy data. The reconstruction by the ASD algorithm. In the cross-sections thick ('red' in color) and thin ('blue' in color) lines show the true signal and the reconstructions, respectively. The visual quality of the reconstructions is poor: artifacts and noise are well seen. 
SPAR, AMPL, RMSE $=0.00369$
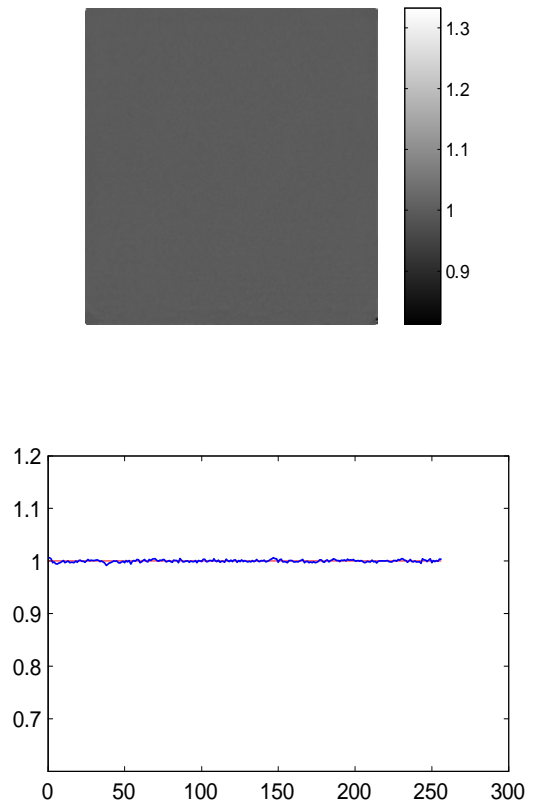

SPAR, PHASE, RMSE $=0.0183$
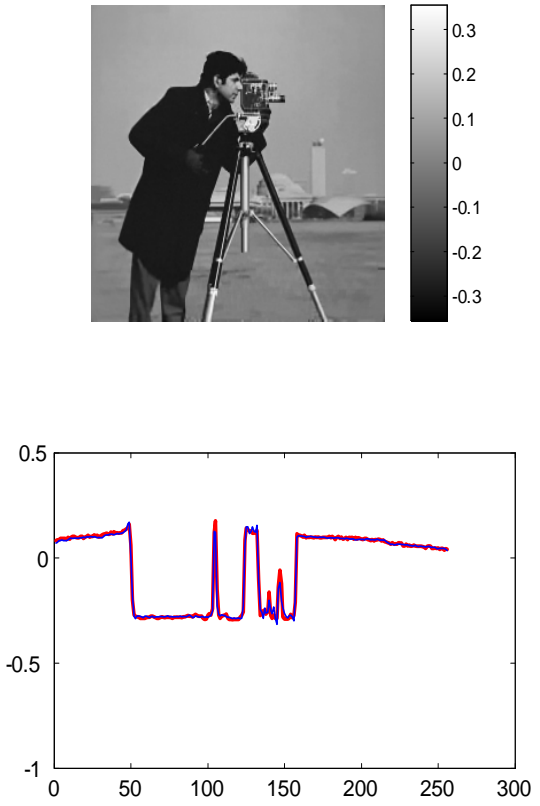

Fig. 6. The phase modulation by the gray-scale cameraman test-image, noisy data. The reconstruction by the proposed SPAR algorithm. RMSE values for the phase and the amplitude are shown at the top. The visual quality of the reconstruction is quite good: wiggles, waves, other artifacts and noisy are nearly wiped out. The noise level in the reconstructions is quite low in comparison with what is achieved in Fig.5. 

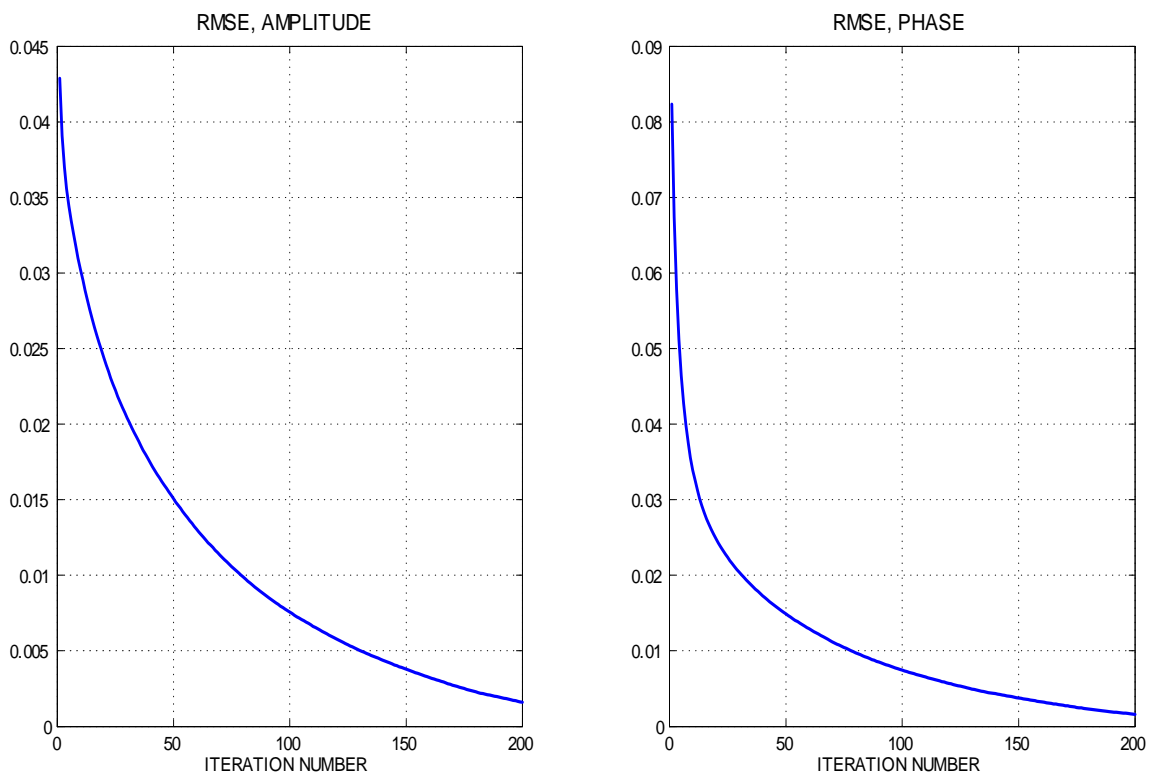

Fig. 7. RMSE for the amplitude and phase reconstructions by the proposed SPAR algorithm versus the number of iterations. The phase modulation object with the binary phase (chessboard test-image), noiseless data. 